\section{$\longrightarrow$ \\ Will non-invasive testing replace liver biopsy in the diagnosis and follow-up of non-alcoholic steatohepatitis (NASH)?}

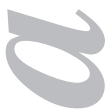
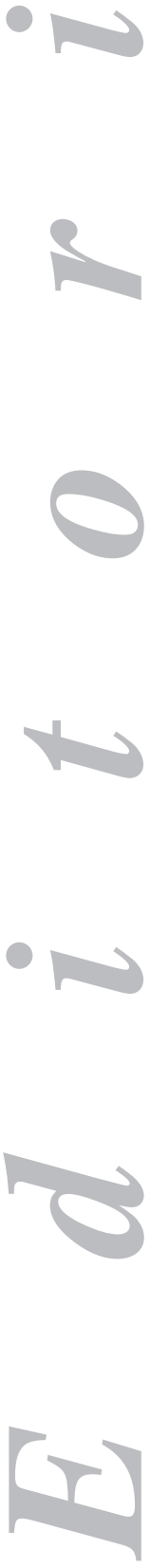

Non-alcoholic steatohepatitis (NASH) is a condition characterized by histological changes similar to those of alcoholic hepatitis in subjects with negligible or nil alcohol use - less than $140 \mathrm{~g}$ of ethanol per week for males and $70 \mathrm{~g}$ for females. NASH should be currently included in non-alcoholic fatty liver disease (NAFLD), which progresses from steatosis to cirrhosis and hepatocellular carcinoma (1).

The growing interest in NASH during the last few years results from a number of reasons. First comes its high prevalence - it is considered the most common cause of hypertransaminasemia, hence the most common liver condition. In the USA 30\% of the adult general population are believed to have NASH, as well as nearly $9 \%$ of the pediatric population. Patients with central obesity and metabolic syndrome are most likely to develop NASH; as a result, prevalence is particularly high in patients with morbid obesity, who may have this condition in up to $95 \%$ of cases. Of these, 20 to $35 \%$ will exhibit NASH signs, and $3 \%$ cirrhosis. These percentages are similar to those reported by Díez-Rodríguez et al. in this issue (2).

The second reason is that fat deposition in the liver has been shown to be not fully harmless, which confirms research by Hans Popper, who back in the sixties stated: "Prolonged fatty transformation of the liver, whether experimental or clinical, commonly leads to fibrous, cirrhotic changes" (3). Despite this statement most authors, based at least partly on the fact that liver function test abnormalities are usually subdued in NASH, considered that simple steatosis was a relatively benign condition. As a result of the Cleveland Clinic findings, which demonstrated that $4 \%$ of patients with simple steatosis develop cirrhosis, fatty liver infiltration is now unanimously considered to be not always a benign process (4).

Histological examination of the liver provides the most accurate information for diagnosing NASH, as it allows to establish inflammation severity and fibrosis extent, and may predict progression to cirrhosis. In fact, based on Brunt's classification, a scoring system is now established to assess steatosis extent, inflammation, and fibrosis stage (5). However, we should bear in mind that liver biopsy also has several shortcomings; it is an invasive, often painful approach with a complication rate of around $0.5 \%$ and significant sampling errors (6). A recent study showed that a single biopsy may not rule out fibrosis in 35\% of cases. Furthermore, steatosis may be absent in advanced disease, hence many authors consider that most cryptogenetic cirrhoses originate in NASH (7). Issues posed by repeat biopsy add to the aforementioned limitations, which makes it virtually impossible to perform longitudinal studies with histological control and a relevant number of subjects.

In the last few years many papers have investigated, particularly in the setting of hepatitis $\mathrm{C}$, the usefulness of alternative, non-invasive tests amenable to regular repetition over time, and providing information on fibrosis stage and liver disease pro- 
gression (8). The increasing relevance of NAFLD has led to use such tests, or to introduce novel ones, in an attempt to differentiate simple steatosis from NASH, and to assess fibrosis stage, both key factors for NAFLD prognosis. Clinical and biochemical variables most commonly associated with advanced fibrosis in NAFLD include diabetes, metabolic syndrome, insulin resistance, elevated AST/ALT index, and decreased platelet numbers; however, the use of these parameters alone is of little value in differentiating simple steatosis from NASH (9). This is also the case with conventional imaging techniques. Indeed, the usefulness of ultrasonography, computed tomography, and magnetic resonance imaging is limited for predicting disease stage. This is why more accurate imaging tests and clinico-biological indices are necessary to differentiate simple steatosis, steatohepatitis, and fibrosis extents. Among the former, elastography by FibroScan is most useful to quantitate liver fibrosis; however, it has specific limitations for NASH, particularly in obese subjects. In a recent study of children with NASH FibroScan allowed to differentiate absent fibrosis from advanced fibrosis, but could not identify intermediate stages (10).

Regarding clinico-biological tests, some attempt to differentiate simple steatosis from NASH whereas others aim to assess fibrosis stage. The former include at least three proposals: NASH test, Palekar index, and Shimada index. These combine age, body mass index, AST/ALT ratio, hyaluronic acid or adiponectin, serum collagen type 4 leves, and HOMA index (8). Some authors have devoted their research to diagnosing NASH in patients with morbid obesity. In this respect, a recent multivariate analysis at the University of San Francisco found six factors predictive for NASH. These include hypertension, diabetes, sleep apnea, ALT > 27 IU, AST > 27 IU, and race other than black (11).

Many more studies have assessed fibrosis staging, to the extent that the number of papers in this field is overwhelming. Nevertheless, many of them lack adequate confirmation in multicenter trials. Biological parameters under study include indirect markers, which reflect liver changes but are not directly related to extracellular matrix (ECM) metabolism, and direct markers of changes occurring in the ECM. Most fibrosis indices are based on mathematical models that combine serum markers, both direct and indirect, and clinical data (12). Given the significance of apoptosis in the pathogenesis of NASH, the levels of fragments generated by caspase-3 on targeting cytokeratin-18 (CK-18) have been proposed as an apoptosis marker and an activity/severity index for NASH (13). Genomic and proteomic techniques to investigate the thousands of genes and proteins involved in the pathogenesis of fibrosis are also being assessed; however, their cost and challenging interpretation represent a serious barrier to clinical use. Methods predictive of fibrosis that have been used in the analysis of extensive series of patients with NAFLD include "BARD", which uses the sum of three simple variables (body mass index, ALT/AST ratio, and diabetes) to identify patients with NAFLD and advanced fibrosis. Another method is "OELF" (Original European Liver Fibrosis), which has been used for a number of liver conditions including $\mathrm{NASH}$, and combines the following parameters: age, hyaluronic acid, PIIIP, and tissue metalloproteinase inhibitor I. A simplified version is designated "ELF" (14). The system proposed by Angulo, known as "NFS", is one of the most commonly used. It has been applied to large series of patients, and comparative studies versus other tests such as Fibrometer and APRI have been carried out. NFS includes six variables: age, body mass index, diabetes, AST/ALT ratio, platelets, and albumin. A mathematical formula is applied to these parameters by which two cutoff points are established allowing to tell patients with no fibrosis 
from those with advanced fibrosis (15). It is this system by Angulo that Díaz-Rodríguez et al. used in their study of patients with morbid obesity. Their paper discusses a well-designed study comparing fibrosis extent in liver biopsies obtained during bariatric surgery versus NFS. The study has definite strengths, including the availability of biopsies for each patient, which significantly reduces potential sampling errors, and that changes in fibrosis were acknowledged with NFS in the second study, some time after bariatric surgery. The study also has a number of limitations - most important of all is the short series of patients, whereby the number of subjects included in each fibrosis stage, particularly for advanced stages, is very small, which forced the authors to pool patients together.

Finally, we must consider the role to be played by non-invasive tests in the management of patients with NAFLD. There is no doubt that combining imaging techniques and clinico-biological testing will prove particularly useful for longitudinal studies providing information on disease progression, as well as of great help in managing these patients. Most challenging for non-invasive test selection is the huge plethora of assays overwhelming the literature, whose results are difficult to compare. When deciding which tests will be used the following key points should be considered: power to differentiate simple steatosis from steatohepatitis, and to distinguish intermediate fibrosis stages (not only advanced fibrosis), which will document progression capability (16). The most important thing to know is whether a combination of imaging techniques and clinico-biological tests will ultimately avoid liver biopsy, or simply help us decide on its indication and proper timing. I think there is currently no clear answer to this question, and many more studies will be needed before reaching a definite conclusion. We may here recall Plinio's statement in the Prologue to his Natural History: "It is a difficult task to give novelty to what is old, authority to what is new" (17).

\section{F. Pons Romero}

Service of Gastroenterology and Hepatology. Hospital Universitario Marqués de Valdecilla. Santander, Spain

\section{REFERENCES}

1. Ludwig J, Viggiano TR, McGill DB, Ott BJ. Nonalcoholic steatohepatitis. Mayo Clinic experiences with a hitherto unnamed disease. Mayo Clinic Proc 1980; 55: 434-8.

2. Díez Rodríguez R, Ballesteros Pomar MD, Calleja Fernández A, González de Francisco T, Olcoz Goñi JL. Usefulness of an index score as a predictor of hepatic fibrosis in obese patients undergoing bariatric surgery. Rev Esp Enferm Digest 2009; 101: 528-35.

3. Popper P, Schaffner F. El hígado su estructura y función. Cap. 26. Barcelona: Edt. Noguer; 1962.

4. Matteoni CA, Younossi ZM, Gramlich T, Boparai N, Liu YC, Mc Cullough AJ. Non-alcoholic fatty liver disease: a spectrum of clinical and pathological severity. Gastroenterology 1999; 116: 1413-9.

5. Brunt EM, Jannay CG, Di Bisceglie AM, Neuschwander-Tetri BA, Bacon BR. Non alcoholic steatohepatitis: a proposal for grading and staging the histological lesion. Am J Gastroenterol 1999; 94: 2467-74.

6. Costera L, Negre I, Samii K, Buffet C. Pain experienced during percutaneous liver biopsy. Hepatology 2000; 32: 477-81.

7. Ratziu V, Charlotte F, Heurtier A Gombert S, Giralt P, Brucker E. Sampling variability of liver biopsy in non alcoholic fatty liver disease. Gastroenterology 2005; 128: 1898-906.

8. Castera L. Non invasive diagnosis of steatohepatitis and fibrosis. Diabetes and metabolism 2008; 34: 674-9.

9. Crespo J, Fernández-Gil P, Hernández-Guerra M, Cayón A, Mayorga M, Domínguez-Díez A, et al. Are there any predictive factors of severe liver fibrosis in morbid obese patients with nonalcoholic steatohepatitis? Obes Surg 2001; 11: 254-7.

10. Nobili V, Vizzutti F, Arena U, Abraldes JG, Marra F, Pietrobattista A, et al. Accuracy and reproducibility of transient elastography for the diagnosis of fibrosis in pediatric nonalcoholic steatohepatitis. Hepatology 2008; 48: 442-8. 
11. Campos GM, Bambha K, Vittnghoff E, Rabl C, Posselt AM, Ciovica R, et al. A clinical scores system for predicting non alcoholic steatohepatitis in morbid obese patients. Hepatology 2008; 47: 1916-23.

12. Vuppalanchi R, Chalasani N. Non alcoholic fatty liver disease and non alcoholic steataohepatitis: selected practical issue in their evaluation and management. Hepatology 2009; 49: 306-17.

13. Wieckowska A, Zein N, Verien LM. In vivo assessment of liver apoptosis as novel biomarkers of disease severity in non alcoholic fatty liver. Hepatology 2006; 44: 27.

14. Cales P, Laine F, Boursier J, Deugnier Y, Moal V, Oberti F, et al. Comparison of blood test for liver fibrosis specific or not to NAFLD. J Hepatol 2009; 50: 165-73.

15. Angulo P, Hui JM, Marchesini G, Bugianesi E, George J, Farrell GC, et al. The NAFLD fibrosis score: a noninvasive system that identifies liver fibrosis in patients with NAFLD. Hepatology 2007; 45: 846-54.

16. Hashimoto E, Farrell GC. Will non invasive markers replace liver biopsy for diagnosing and staging fibrosis in non alcoholic steatohepatitis. J Gastroenterol Hepatol 2009; 24: 564-8.

17. Plinio Cayo Segundo. Historia natural. Prólogo dedicado a Tito Flavio Vespasiano. Trad G. Huerta. Madrid; 1624. 